\title{
El examen físico: ¿Para qué, Cómo, Cuándo y a Quiénes hacerlo?
}

\section{Introducción}

Mathieu, Chauffard, Glénard y Gilbert; Guyón, Israel y Belington; Rualt, Neumann y Hoover. La lista precedente no corresponde a un equipo de fútbol, tampoco al comité editorial de nuestra publicación. Se trata de maniobras de palpación del hígado, riñón, y evaluación de la expansión torácica respiratoria, respectivamente. El examen físico es aprendido por el estudiante de medicina con un alto grado de automatización. Durante nuestra época de estudiantes, probablemente en más de una ocasión, hemos pasado momentos de angustia intentando retener y reproducir estas maniobras. La práctica cotidiana nos indicaría que la importancia de las mismas en la actualidad es limitada. ¿Será esto debido a que el examen físico en sí, es de utilidad relativa? ¿Será porque la aparatología moderna, que supera con creces la sensibilidad y especificidad de las maniobras semiológicas, las ha vuelto obsoletas? En todo caso, ¿debemos siempre examinar a nuestros pacientes, aún en el contexto de un control de salud? El examen físico, ¿debe siempre ser completo o sólo algunas maniobras serían de utilidad en el paciente asintomático?

A continuación analizaremos al exámen físico desde el punto de vista de sus objetivos, precisión diagnóstica, alcances y limitaciones para poder luego reflexionar sobre su utilización como herramienta en nuestra actividad asistencial.

\section{¿Para qué realizamos el examen físico?}

Examinamos a nuestros pacientes con dos tipos de fines:

1 - Diagnósticos: en este sentido el examen físico es una prueba diagnóstica más, y cabe analizarlo bajo las mismas consideraciones en cuanto a sensibilidad, especificidad y valores predictivos de cada maniobra. Sin embargo, se diferencia de otros métodos auxiliares en que las maniobras tienen muy baja morbilidad (el único riesgo se relaciona con las consecuencias de la detección de falsos positivos).

2 - Desarrollo de la relación médico paciente: existen beneficios no relacionados con objetivos diagnósticos. El solo hecho de la revisación transmite al paciente, en la mayoría de los casos, sensación de cuidado, atención y reaseguro.

\section{¿Qué tan exacto es el examen físico como prueba diagnóstica?} Tenemos información sobre la frecuencia con que un determinado signo aparece en una entidad (sensibilidad), o la probabilidad de que estando ese signo presente, el paciente tenga esa entidad (valor predictivo positivo). Pero rara vez hallamos información acerca de la probabilidad de que un paciente sano no tenga ese signo (especificidad), o la probabilidad de que frente a la ausencia de ese signo el paciente esté sano (valor predictivo negativo).

En general predominan los signos más sensibles que especificos

No obstante, los signos del examen físico son habitualmente poco exactos y no es rara la variabilidad interobservador (baja precisión). Por ejemplo, la auscultación del cuello para el diagnóstico de estenosis carotídea tiene una sensibilidad aproximada de un $60 \%$ y una especificidad del 25 al 60\%. En general el examen físico es más preciso para el diagnóstico de síndromes que para el de entidades (síndrome de condensación vs. cáncer de pulmón).

Para confirmar un diagnóstico intentaremos incrementar fundamentalmente la especificidad de nuestro examen físico, para lograr así el mayor valor predictivo positivo posible.

Para descartar un diagnóstico deberemos optimizar la sensibilidad por ser ésta la propiedad más importante para determinar el valor predictivo negativo de una prueba diagnóstica (1).

Cuando un signo tiene una especificidad extremadamente alta (mayor del $95 \%$ ), su presencia tiende a asegurar la presencia de la condición investigada. En estos casos decimos que dicho signo es patognomónico. Cuando un signo tiene una sensibilidad extremadamente alta, su ausencia tiende a descartar la presencia de la condición investigada. Veamos algunos ejemplos:
Signos patognomónicos o extremadamente específicos

\begin{tabular}{llc}
\hline Condición investigada & \multicolumn{1}{c}{ Signo } & Especificidad \\
\hline Enfermedad de Wilson & Anillo de Kaiser-Fleischer & $100 \%$ \\
\hline Derrame pleural & Percusión-Auscultación sugestiva & $100 \%$ \\
\hline Melanoma & Características clínicas de la lesión & $96-99 \%$ \\
\hline
\end{tabular}

Signos extremadamente sensibles

\begin{tabular}{llc}
\hline Condición investigada & \multicolumn{1}{c}{ Signo } & Especificidad \\
\hline Hipertensión endocraneana & Pérdida de pulsación venosa & \\
& retinal espontánea & $100 \%$ \\
\hline Insuficiencia cardíaca & Taquicardia & $98 \%$ \\
\hline Derrame pleural & Percusión-Auscultación sugestiva & $96 \%$ \\
\hline
\end{tabular}

Pero en la mayoría de los casos no tenemos la posibilidad de contar con signos tan categóricos (100\% sensibles o 100\% específicos), por lo que automáticamente buscaremos la presencia 0 ausencia de varios signos que en conjunto aumenten o disminuyan la probabilidad de presencia o ausencia de determinada condición. Cuanto menor sea la interdependencia entre sí de los distintos signos considerados, mayor será el aumento de exactitud (si los signos son interdependientes, este proceso no tendría mayor utilidad pues no adicionarían información unos a otros). Ej.: ingurgitación yugular y congestión hepática, representan consecuencias de un mismo fenómeno (dificultad en el retorno venoso) y por lo tanto no deberían tomarse como aditivos en la presunción de insuficiencia cardíaca. Pero cualquiera de ellos más la auscultación de tercer ruido cardíaco sí aumentan la probabilidad postest, pues responden a mecanismos fisiopatológicos independientes.

\section{CUADRO RESUMEN}

\section{Características del exámen físico como prueba diagnóstica}

* Predominan los signos más sensibles que específicos.

* La exactitud de una misma maniobra o signo es diferente para distintas entidades.

* Las maniobras y signos del exámen físico son en general poco exac tos

* La exactitud del examen físico se aumenta automáticamente al generar nuevas maniobras (búsqueda de más signos relacionados con la condición investigada).

* Por medio del interrogatorio es posible aumentar la probabilidad previa y de esta manera incrementar el rendimiento de una maniobra. Ej: criterios sugestivos de hipertensión renovascular en el interrogatorio aumentan el valor predictivo positivo del soplo abdominal.

* Existe un alto grado de dependencia entre diferentes signos del exámen físico.

* No es infrecuente el desacuerdo clínico en el exámen físico (baja precisión).

* Como herramienta diagnóstica es menos poderoso que el interrogatorio pero es más importante que muchas otras pruebas.

¿Qué errores podemos cometer utilizando al exámen físico como herramienta diagnóstica?

1- Descartar un diagnóstico probable por no hallar algún signo que es poco sensible.

Ej.: paciente de 35 años sin factores de riesgo coronario, con dolor precordial que aumenta con la inspiración, la tos y los cambios posturales, en el que se descarta pericarditis aguda por no hallarse frote pericárdico (el frote es poco sensible para el diagnóstico de pericarditis). 
2- Considerar como primera hipótesis diagnóstica una entidad, a pesar de la ausencia de signos muy sensibles del exámen físico.

Ej.: mujer de 35 años con leve pérdida de peso, irritabilidad y taquicardia, a la que no se le palpa la tiroides, a pesar de lo cual se piensa en primer lugar en hipertiroidismo (95\% de los casos tienen bocio, por lo que el no tenerlo casi descarta el hipertiroidismo).

\section{3- Presumir un diagnóstico poco probable con el hallazgo de signos poco específicos.}

Ej.: paciente de 19 años con astenia y fiebre de 12 días de evolución en el que se presume en primer lugar linfoma, por la presencia de adenopatías cervicales.

4- No considerar como primer diagnóstico una entidad a pesar de la presencia de signos específicos en el exámen físico.

Ej.: paciente de 17 años con cefalea bitemporal y occipital y papiledema bilateral (alta especificidad) en el que no se considera masa cerebral, por no tener signos de foco neurológico.

5- Considerar un aumento importante de la probabilidad de un diagnóstico por la adición de signos con alto grado de dependencia.

Ej.: edemas, ingurgitación yugular y rales crepitantes en la insuficiencia cardíaca congestiva.

Ejemplos de especificidad y sensibilidad de maniobras y signos del exámen físico (Referencias 2 a 8)

\begin{tabular}{|c|c|c|c|}
\hline Condición investigada & Signo & Sensib. & Especif. \\
\hline Ascitis & Onda ascítica fluída & $53 \%$ & $92 \%$ \\
\hline Ascitis & Abultamiento de flancos & $94 \%$ & $30 \%$ \\
\hline Esplenomegalia & $\begin{array}{l}\text { Matidez del Traube } \\
\text { (método de Nixon) }\end{array}$ & $62 \%$ & $72 \%$ \\
\hline Esplenomegalia & $\begin{array}{l}\text { Palpación } \\
\text { (global de todas maniobras) }\end{array}$ & $58 \%$ & $94 \%$ \\
\hline Hepatomegalia & $\begin{array}{l}\text { Palpación } \\
\text { (global de todas maniobras) }\end{array}$ & $46 \%$ & $63 \%$ \\
\hline $\begin{array}{l}\text { Vértigo de origen } \\
\text { periférico }\end{array}$ & Maniobra de Barany & $40-60 \%$ & $94 \%$ \\
\hline Insuficiencia cardíaca & Reflujo abdominoyugular & $30-60 \%$ & $94 \%$ \\
\hline Sinusitis & Compresión de senos maxilares & $48 \%$ & $62 \%$ \\
\hline Sinusitis & Transiluminación & $76 \%$ & $48 \%$ \\
\hline Podagra & $\begin{array}{l}\text { Características clínicas } \\
\text { de la lesión }\end{array}$ & $70 \%$ & $82 \%$ \\
\hline
\end{tabular}

\section{Utilidad del examen físico}

Ya hemos comentado los beneficios del examen físico en el desarrollo de la relación médico-paciente. En este sentido el examen físico es a menudo un acto esencial en la entrevista médica. Esto se relaciona fuertemente con las expectativas del paciente.

Para conocer las mismas, realizamos una encuesta entre 50 empleados de una empresa (ninguno de ellos era médico) consistente en las siguientes preguntas:

1. ¿Considera usted que cuando consulta al médico por un problema determinado de salud, el exámen físico es imprescindible?
a) SI
b) NO

2. ¿Considera usted que cuando consulta al médico por un control de rutina, el exámen físico es imprescindible?
a) SI
b) NO

3. Si cree necesario al exámen físico en un control de rutina, ¿qué maniobras considera que deberían formar parte de la revisación?

\section{Resultados}

\begin{tabular}{lcccc}
\hline Item & Si & No & Veces citado & Porcent. \\
\hline EF* imprescindible en problema de salud & 44 & 6 & & $88 \%$ \\
\hline EF* imprescindible en control de rutina & 37 & 13 & & $74 \%$ \\
\hline Maniobras del EF* en control de rutina: & & & \\
\hline Registro de Presión Arterial & & 27 & $54 \%$ \\
\hline Auscultación Cardíaca & & 25 & $50 \%$ \\
\hline Auscultación Pulmonar & & 21 & $42 \%$ \\
\hline Reflejos & 17 & $34 \%$ \\
\hline Exámen oftalmológico (fondo de ojo) & 8 & $16 \%$ \\
\hline Palpación de abdomen & 8 & $16 \%$ \\
\hline Discrecional del médico & 7 & $14 \%$ \\
\hline Palpación del Pulso & 3 & $6 \%$ \\
\hline Peso & 3 & $6 \%$ \\
\hline Palpación de ganglios & 3 & $6 \%$ \\
\hline Palpación de mamas & & 3 & $6 \%$ \\
\hline Exámen de oídos (otoscopía) & & 3 & $6 \%$ \\
\hline Registro de temperatura & & 2 & $4 \%$ \\
\hline Inspección de fauces & & 2 & $4 \%$ \\
\hline
\end{tabular}

* EF: exámen fisico

Como vemos, la gran mayoría de los pacientes siente que es imprescindible el examen físico en toda visita médica, incluso cuando no consultan por un síntoma específico, sino por un control de rutina. Además aproximadamente la mitad de los mismos identifica como maniobras necesarias durante un control de salud, a la toma de presión arterial y la auscultación cardiopulmonar. Solo un 14\% de los encuestados deja enteramente al criterio médico las maniobras del examen físico necesarias, sin mayores expectativas previas.

En cuanto a los médicos, una encuesta llevada a cabo en USA, reveló que el $90 \%$ de los profesionales encuestados (médicos de familia) consideraba necesario un metódico examen físico como parte del control periódico de salud, realizando durante el mismo un promedio de 11 a 14 maniobras dependiendo de la edad y sexo del paciente (9). Por otro lado, en cuanto a su utilidad como prueba diagnóstica, si bien el examen físico es en general poco exacto, existen casos en que puede superar a otros métodos auxiliares modernos y costosos. Existen estudios en que la exactitud diagnóstica de la palpación de cuello para la pesquisa de metástasis cervicales de carcinoma escamoso orofaríngeo fue superior a la de la tomografía computada ( $66 \%$ vs. $62 \%$ ) superándola tanto en sensibilidad como en el valor predictivo positivo (10). Del mismo modo, el examen físico es tan confiable como la arteriografía o la ecografía en la detección de lesiones vasculares cervicales por heridas penetrantes en la zona II del cuello, que requieren cirugía reparadora (11). También, en manos experimentadas, la semiología alcanza una excelente sensibilidad y especificidad ( $90 \%$ y $99 \%$ respectivamente) en el diagnóstico de lesiones del ligamento cruzado posterior (12). La impresión del estado general junto a la evaluación del esfuerzo respiratorio y la interacción con el medio, superan el 90\% de exactitud para el diagnóstico de hipoxemia (saturación por oximetría de pulso <95\%) en niños menores de 1 año (13). En pacientes asintomáticos, la auscultación cardíaca tiene una sensibilidad del $70 \%$, una especificidad del $98 \%$ y valores predictivos positivo y negativo del $92 \%$ para el diagnóstico de enfermedad valvular, tornando innecesaria a la ecocardiografía como método de rastreo (14). Sandler encontró que en el $56 \%$ de los casos se llega al diagnóstico correcto mediante el interrogatorio y que el examen físico llevaría esa cifra al $73 \%$, mientras que el resto de las pruebas diagnósticas tienen un porcentaje de contribución diagnóstica mucho menor. Pero más allá de la sospecha clínica y a la hora de la corroboración diagnóstica, el examen físico, como dijimos, tiene en general una exactitud limitada y frecuentemente una baja reproducibilidad interobservador. Por lo tanto, sus hallazgos, más sensibles que específicos, son más a menudo instrumentos inductores de otras pruebas de confirmación. Por eso es importante una utilización e interpretación racional del examen físico, para no generar cascadas diagnósticas innecesarias, mu- 
chas veces alarmantes y costosas para el paciente.

En el paciente asintomático, solo algunas maniobras están respaldadas por la evidencia científica como de utilidad para el rastreo de condiciones asintomáticas u oligosintomáticas.

Recomendaciones para la pesquisa a través del examen físico en pacientes adultos asintomáticos. (15)

\begin{tabular}{lccl}
\hline Procedimiento & $\begin{array}{c}\text { Grado de } \\
\text { evidencia }\end{array}$ & Edad & Frecuencia \\
\hline Controlde la TA & I & & cada 2 años \\
\hline Exámen dental & I & & anualmente \\
\hline Palpación mamaria en mujeres & I & & anualmente \\
\hline Auscultación cardíaca & II y III & $20-59$ & una vez \\
\hline Exámen de la piel & III & 20-59 & una vez \\
\hline Control delpeso & III & & cada 4 años \\
\hline Agudeza visual & III & $>$ de 60 & anualmente \\
\hline Audición & III & $>$ de 60 & anualmente \\
\hline $\begin{array}{l}\text { Palpación de abdomen } \\
\text { por aneurisma de Aorta }\end{array}$ & III & $>$ de 60 & anualmente \\
\hline
\end{tabular}

\section{Conclusiones}

El examen físico es una herramienta de gran valor para el médico y cumple un doble propósito: desarrollar la relación médico-paciente y como prueba diagnóstica. La gran mayoría de los pacientes, de hecho lo ven como parte imprescindible de la entrevista médica, sintiéndose insuficientemente atendidos si no está presente, aún cuando el mismo no aporte, desde un punto de vista estrictamente científico, mayor información ni plantee cambios en la conducta terapéutica. Incluso es probable que si la revisación no incluye el registro de la presión arterial y la auscultación cardíaca y pulmonar, la sensación de cuidados insatisfechos persista, pues son estas maniobras las identificadas como parte de una consulta médica completa, y solo una minoría de los pacientes deja enteramente a criterio del médico las maniobras del examen físico necesarias de acuerdo a las circunstancias. Podríamos concluir en este punto que el examen físico debería formar parte de toda consulta médica, por supuesto incluyendo las maniobras recomendadas para el rastreo de condiciones silentes según edad y sexo cuando se trata de un control de salud, y las maniobras específicas de exploración requeridas según cada caso cuando el paciente consulta por determinados síntomas. Existen pacientes reticentes al contacto físico, pero éstos suelen expresarlo directa o indirectamente durante la entrevista. En estos casos, solo se realizará el examen físico si nos puede ser de utilidad como instrumento diagnóstico. Enfocando al examen físico como mera prueba diagnóstica, si bien es cierto que en líneas generales es poco exacto, combinando varias maniobras y a partir de un ordenado interrogatorio, logramos más de las $3 / 4$ partes de los diagnósticos a los que arribamos. En conjunto, los métodos auxiliares no reemplazan a un adecuado examen físico por más sofisticados y modernos que fueren. Existen maniobras de utilidad cuestionable; así la palpación del hígado no aportó mayor precisión diagnóstica en cuanto al volumen real del órgano (que es pronóstico en la cirrosis) (16). Sin embargo, no deberíamos abandonar el aprendizaje de un adecuado y metódico exámen físico pues si bien el conocimiento para convertirse en sabiduría requiere de experiencia, no menos cierto es que la experiencia, para desarrollarse, se sustenta en el conocimiento, y la provechosa utilización del examen físico no es la excepción (17). Lo central es el manejo racional de la información que uno obtiene con la revisación de sus pacientes. Tener claro de antemano el propósito de nuestras maniobras, lo que estamos buscando y los alcances y limitaciones de los hallazgos. En los pacientes asintomáticos, el examen físico debería evolucionar de una mera recolección de datos mediante una revisación de los pies a la cabeza "para ver si aparece algo alterado", a un esfuerzo evaluativo del paciente con un sentido racional y direccionado.

\section{Dr. Alejandro Regueiro}

Sistema de Protección Médica

\section{Referencias}

1. Lamb MG, Scheller JM. The rational clinical examination. JAMA 1992 0ct 28; 268 (16):2164-5.

2. Cummings S, et al. The predictive value of physical examination for ascites. West J Med. 1985 May; 142 (5):633-636.

3. Grover SA, et al. Does this patient have splenomegaly? JAMA 1993 Nov 10; 270 (18): 2218-21.

4. Williams JW Jr, et al. Does this patient have sinisitis? Diagnosing acute sinusitis by history and physical examination. JAMA 1993 Sep 8; 270 (10): $1242-46$.

5. Naylor CD. Physical examination of the liver. JAMA 1994 Jun 15; 271 (23): 1859-65.

6. Froehling DA, et al. Does this dizzy patient have a serious form of vertigo? JAMA.1994 Feb 2; 271 (5):385-8.

7. Siminoski K.Does this patient have a goiter? JAMA 1995 Mar 8; $273(10): 813-7$.

8. Whited JD, et al. Does this patient have a mole or a melanoma? JAMA 1998 Mar 4; 279 (9): 696-701.

9. Luckmann R, Melville SK. Periodic Health Evaluation of adults: a survey of family physicians. J Fam Pract 1995 Jun; 40 (6): $547-54$.

10. August M, Nguyen M. Evaluation of metastatic neck disease by compyted tomography. Int J Oral Maxillofac Surg 1994 0ct; 23 (5): $290-3$.

11. Atteberry LR, Dennis JW, Menawat SS, et al. Physical Examination alone is safe and accurate for evaluation of vascular injuries in penetrating Zone II neck trauma. J Am Coll Surg 1994 Dec; $179(6): 657-62$

12. Rubinstein RA Jr, Shelbourne KD, Mc Carroll JR, et al. The accuracy of the clinical examination in the setting of posterior cruciate ligament injuries. Am J Sports Med 1994 JuL-Aug; 22 (4): $550-7$. 13. Margolis PA, Ferkol TW, Marsocci S, et al. Accuracy of the clinical examination in detecting hipoxemia in infants with respiratory illness. J Pediatr 1994 Apr; 124 (4): $552-60$.

14. Roldan CA, Shively BK, Crawford MH. Value of the cardiovascular physical examination for detecting valvular heart disease in asymptomatic subjects. Am J Cardiol 1996 Jun 15; 77 (15): $1327-31$

15. Regueiro A, Velazquez A. Prevención en La Práctica Clínica. En: PROFAM: Programa de Educación a Distancia de Medicina Familiar y Ambulatoria / Rubinstein E, dir; Caffaro de Hernández

N, coord.didáctica / Fundación M.F.- Medicina Familiar para el Desarrollo de la Medicina Familiar y la Atención Primaria de la Salud. 1ra. ed. Buenos Aires: Hosp. Italiano de Buenos Aires. Unidad de Medicina Familiar y Preventiva; Dep. de Docencia e Investigación, c1997. v.2, p. 41-88.

16. Zoli M, Magalotti D, Grimaldi M, et al. Physical examination of the liver: is it still worth it? Am J Gastroenterol 1995 Sep; 90 (9): $1428-32$.

17. Sharma OP. Symptoms and signs in pulmonary medicine: old observations and new interpretations. Dis Mon 1995 Sep; 41 (9): $577-638$.

\section{Bibliografia general}

* U.S. Preventive Services Task Force. Guide to Clinical Preventive Services. Baltimore: Williams \& Wilkins,1996

* Fullar E, et al. Promoting prevention in primary care: controlled trial of low tecnology, low cost approach. Br Med J 1987; 294: 1080-2

*Sackett DL, et al. Epidemiología clínica. Ciencia básica para la medicina clínica.2da. ed. Buenos Aires. Panamericana, 1994

*The Periodic Health Examination 2. 1985 update. Canadian Task Force on the Periodic Health Examination. Can Med Assoc ]; 1986: 134: $724-7$

* Oboler SK, et al. The Periodic Physical Examination in Asymptomatic Adults. Ann Int Med 1989;110: 214-26

*Hayward RS, et al. Preventive Care Guidelines: 1991. Ann Int Med 1991; 114: 758-3

*Sox HC, Jr. Preventive Health Services in Adults. N Engl J Med 1994; 330: 1589-95. 